\title{
The Basic Situation and Analysis of Shadow Banking in China and Foreign Countries
}

\author{
Zhang Hao \\ Economics School, Shanghai University, Shanghai, China
}

\section{Email address:}

zhangles_90@126.com

\section{To cite this article:}

Zhang Hao. The Basic Situation and Analysis of Shadow Banking in China and Foreign Countries. International Journal of Economics, Finance and Management Sciences. Vol. 3, No. 5, 2015, pp. 635-640. doi: 10.11648/j.ijefm.20150305.39

\begin{abstract}
Shadow banking was first put forward by Mc Cully, the executive director of Pacific Investment Management Company, in the Fed's annual meeting in 2007, which he defines shadow banking as a kind of financial entities that divorced from the traditional banking system, almost unregulated or less regulated. Since then, the concept of shadow banking spread rapidly worldwide, and has been widely cited and elucidated. At the same time, shadow banking has become an increasingly important objective existence in China. Although the scale of shadow banking is still far smaller than the traditional system, but the development of shadow banking in China is fast, and the social influence is widespread. Therefore, on the basis of studying the history and current situation of the shadow banking around the world, this paper analyzes the Chinese shadow banking system.
\end{abstract}

Keywords: Shadow Banking, Interest Rate Marketization, Currency Liquidity

\section{中外影子银行的基本现状与分析}

张昊

经济学院, 上海大学, 上海, 中国

\section{邮箱}

zhangles_90@126.com

摘要：影子银行的说法最早由美国太平洋投资管理公司的执行董事麦卡利在 2007 年的美联储年度会议上提出，他定义 影子银行为游离于传统银行体系之外的，几乎不受监管或受较少监管的金融实体。此后，影子银行的概念在全球范围 内迅速传播, 被广泛地引用和阐发。影子银行在中国也变成越来越重要的客观存在, 虽然规模与银行体系相比还相去 甚远, 但中国影子银行发展速度迅速, 社会影响面广泛。因此本文在研究世界范围内影子银行发展历程与现状的基础 上，对中国的影子银行体系进行了分析。

关键词：影子银行，利率市场化，货币流动性

\section{1. 引言}

流动性松紧的判断, 以新增商业银行信贷量作为衡量 社会整体货币环境宽松度的传统指标, 但由于经济的创新 发展, 市场紧盯的银行存贷款数据已经不能完整的表明整 体经济体资金供求状况。几十年来, 对于流动性的研究,
正统的货币数量理论以基础货币、广义货币供给量和货币 乘数为基础进行分析, 但影子银行的出现和崛起改变了这 一格局, 中央银行和商业银行流动性联动和其资产负债表 的勾稽关系做深入研究的思想被打破。伴随着货币数量论 所描述的流动性创生机制再也不能全面描述流动性, 影子 银行步入了历史的舞台。 
影子银行这一术语最早出现在堪萨斯城联邦储备银 行杰克逊霍尔经济论坛上, 由太平洋投资管理公司的执行 董事麦克雷提出。同时, 他还指出这些不受监管的影子银 行所使用的为自己融资的商业票据没有担保, 但真正的银 行对于此类票据却可以提供流动性额度支持。在商业票据 投资者可能拒绝票据展期的情况下, 影子银行比较容易遭 受及挤兄危机, 从而陷入流动性困境。此后, 债券大王格 罗斯在题为 “关注影子银行体系” 的专栏文章中谈到, 影 子银行体系等同于现代金融体系, 因为它囊括了二战后商 业银行体系以外的几乎所有的金融创新。影子银行的说法 自此传播开来，被广泛使用。

同时, 金融危机的爆发使影子银行受到了更加高度的 关注。然而截止到目前, “影子银行” 还没有公认的统一 定义。这一方面与该术语出现时间较短有关, 另一方面也 与不同经济实体的金融架构及监管方式有关。此外, 影子 银行体系随着金融创新和监管体制的变化而变化, 因此具 有不固定性和动态演变特征。但对于其范围的界定, 各方 定义中都强调了 “发挥着类似商业银行的基本功能, 却不 受监管或仅受较少监管”的共同特征。

\section{2. 影子银行的发展与特征}

\section{1. 影子银行的演进}

影子银行是一个新兴的概念, 但影子银行的存在却已 经有上百年的历史, 甚至可以说, 影子银行的历史与商业 银行一样长。影子银行的演进发展可以划分为四个阶段。

第一个阶段, 1970年以前, 影子银行形态主要是传统 的信托公司, 规模较小, 业务活动也相对简单。

第二个阶段，1970-1980年间，特征是金融脱媒。伴 随着放松利率管制和金融市场的发展, 金融脱媒逐步兴起, 金融公司、货币市场共同基金及证券化的贷款迅速增加， 商业票据、欧洲债券以及垃圾债券迅速发展, 在这个时期, 影子银行得到一定的发展, 但商业银行、保险公司仍然占 据了美国金融市场的主导地位。

第三个阶段，1980-2000年间，特征是资产证券化。 这个时期, 金融部门核心的工作就是将非市场化的资产转 换为市场化的证券。住房抵押贷款及以后的自助贷款和信 用卡应收账款, 被证券化之后在二级市场上买卖。银行和 存贷机构提供系列服务, 比如发行、服务、持有以及贷款 分配, 其贷款被分销给不同的影子银行机构和大众所持有, 影子银行渗入到金融体系的方方面面。

第四个阶段从上世纪末开始, 特征是高度证券化基础 上的交易衍生化。标志是1999年美国颁布《金融服务现代 化法》, 分业经营退出历史舞台, 投资银行、商业银行、 保险公司、共同基金的边界彻底被打破，纷纷经营起形形 色色的影子业务。更重要的是, 随着金融自由化和全球化, 大量衍生工具如CDS、CDO被疯狂创造并卖到全世界投资者 手中，交易呈几何数量暴增，对冲基金、结构化投资实体 等影子银行大行其道。文化因素在中国对外直接投资 (OFDI) 的区位选择中的作用越来越重要。本文通过对中 国现有的研究文化因素对中国OFDI区位选择影响的文献 进行综述, 发现中国学者对该方向的研究多集中在实证研
究, 且由于选取的衡量文化因素的指标不同, 其分析结果 存在着差异。

\section{2. 影子银行业务的一般特点}

$$
\text { (一) 典型 “三高” }
$$

影子银行业务具有典型的“三高”特点: 高风险偏好、 高杜杆率、高期限错配。

高风险偏好是指, 影子银行由于不受节制的盈利冲动, 往往偏好于高信用风险、低流动性的长期金融资产，这类 资产往往是传统商业银行不愿意或不能涉足的。

高杜杆率有两种含义, 一是资本的杜杆率高, 即大量 利用财务杜杆举债经营, 其总资产与净资本的比率很高, 贝尔斯登、雷曼兄弟的资本杜杆率在破产前的峰值分别达 到了 33.5 倍和 30.7 倍, 其他投行也在 30 倍以上, 而商业银 行一般在 10 倍左右。二是大量利用衍生产品进行高杜杆交 易, 比如购买 $\mathrm{CDS}, \mathrm{CD} 0$ 等高杜杆产品。

高期限错配, 即借短投长, 是影子银行的核心盈利模 式之一，使得流动性风险成为其内生性风险。

（二）较少监管

影子银行从事高风险、高杜杆、高错配的金融业务, 但却受到较少的监管, 获得与银行相当的收益却不承担银 行的成本, 这是典型的监管套利, 导致金融体系风险积累。

需要指出的是, 影子银行并非完全不受监管, 除了金 融危机之前的对冲基金以外，投资银行、金融公司、共同 基金这些影子银行机构都是受到监管的, 只是不受监管当 局对于银行业那样严格的监管。这意味着影子银行无需满 足巴塞尔协议的资本要求, 无需缴纳存款准备金和存款保 险费, 也无需计提贷款拨备, 无需进行大量的信息披露, 从而使得其经营成本降到最低, 利润最大化。

但这只是硬币的一面, 规避监管的同时也意味着影子 银行处于国家金融安全网保护之外, 无法受到央行最后贷 款人或贴现窗口的流动性支持, 投资人无法享受存款保险 的保护, 这也是影子银行的阿喀琉斯之踵。

\section{（三）透明度低}

影子银行的产品结构设计非常复杂, 而且鲜有公开的、 可以披露的信息, 透明度很低。这些金融衍生品交易大都 通过场外交易进行，信息披露制度很不完善。这对监管当 局和投资人都形成了信息 “黑箱” ，也助长了其冒险的激 进行为。

(四) 批发运作

影子银行打破了传统商业银行零售的服务模式, 采取 了类似打包、捆绑、组合的批发销售模式。影子银行不能 像传统的商业银行吸收公众存款以获得融资, 因此通过向 货币市场投资者发行票据、ABCP等货币市场工具或进行回 购交易以获得融资，有时也向证券借贷公司、养老金及保 险公司等中长期投资者发行中期票据及债券, 使用的工具 大多为批发性融资工具。同时，影子银行的投资一般通过 证券市场或者场外衍生产品市场进行, 交易的市场化程度 高于商业银行。

（五）风险传染

与传统的商业银行相比, 影子银行的业务链显得更为 复杂。传统银行的中介业务一般在一家银行内部进行, 但 
影子银行往往透过多家非银行金融机构, 采用复杂的业务 流程来进行融资活动, 将有风险的诸如次级抵押贷款的长 期债务转化为看似无风险的、短期的货币市场工具。复杂 流程将各类影子银行编织成了一个网络, 一旦出现问题, 风险迅速传染，不仅影响到影子银行自身，而且影响到正 规银行体系。

影子银行体系将传统商业银行存款（资金来源）一贷 款（持有到期）的模式转换为金融市场融资-证券化贷款 过程, 从转换过程来看, 其将中长期、低流动性、高风险 的贷款变成了短期、高流动性、低风险的货币市场基金份 额，重要的是货币市场基金拥有更加稳定的资产净值。

\section{3. 全球影子银行的现状与分析}

\section{1. 全球影子银行体系的规模与结构}

若将各种证券化产品和衍生工具也纳入广义流动性 的考虑范围, 即视为影子银行活动的结果, 则根据估算, 2007年末, 全球各种衍生品创造的流动性约占全球广义流 动性的 78\%, 为世界GDP总值的9.6倍; 而广义货币M2仅占 广义流动性的 $10 \%$, 为世界GDP的1.2倍。如用相对狭义的 影子银行概念评估影子银行体系, 我们可借由金融稳定委 员会的最新报告来观察。

金融稳定委员会 (Financial Stablity Board, FSB) 2012年11月18日发布《全球影子银行监测报告 2012 》采用 较保守的评估方法从资金流量表的角度进行分析，对 20 个国家 (阿根廷、澳大利亚、巴西、加拿大、智利、中国、 中国香港、印度、印度尼西亚、日本、韩国、墨西哥、俄 罗斯、沙特阿拉伯、新加坡、瑞士、土耳其、英国、美国） 和欧元区的影子银行体系进行了初步统计, 样本国家和地 区GDP 占全球的 $86 \%$ ，金融市场资产占全球的 $90 \%$ 。统计中 使用其他金融机构资产作为影子银行资产估计的保守代 理指标, 将全部金融机构去掉中央银行、商业银行、保险 公司、养老基金、国有金融机构即为其他金融机构。金融 稳定理事会公布的 20 国集团及欧元区影子银行跟踪报告 数据显示, 2011年末, 以非银行金融机构作为统计对象的 影子银行规模为 67 亿美元, 占受调查国家GDP总额的 $111 \%$ 。
目前, 影子银行占全部金融中介总资产的 $1 / 4$, 约为传统 商业银行规模的一半。

按业务种类划分, FSB推测非股权类基金的规模以 9 万亿美元位居守卫, 而结构性金融工具以 5 万亿美元的规 模紧随其后，占到非银行金融机构总规模的 $10 \%$, 而经纪 业务、金融公司、金融控股公司以及货币市场基金基本上 规模差不多, 各自在 4 万亿美元上下, 分别占到非银行金 融机构规模的 $7 \%$ 。

总体上，影子银行规模占金融业资产的比例大约为 25\%-30\%, 但不同国家存在较大差异。其中, 美国影子银 行规模最大, 虽由 2007 年年底的 27 万亿美元降为 2011 年的 23 万亿美元, 但占比仍到 $35 \%$; 其次是欧元区 22 万亿美元, 占比 $33 \%$; 英国的 9 万亿美元占比升至 $13 \%$; 日本占比降到 $6 \%$ 。

金砖国家的影子银行体系在金融危机后保持了较高 的复合增长率, $\mathrm{FSB}$ 认为中国式增长率最高的国家。FSB 报告显示，自2007年中国的货币市场基金和金融公司两类 影子银行显著增长，增速达 $33 \%$ 。同时评估指出中国 2011 年按非银行金融机构统计的影子银行规模为 0.4 万亿美元, 占所有被统计成员国总量的 $1 \%$ 。

\section{2. 国际影子银行分析}

根据金融稳定委员会对 20 个国家和欧元区的影子银 行体系的初步统计, 全球范围内影子银行图景有以下表现。

\section{2. 1. 商业银行资产总量仍然占优}

若按照金融机构进行划分, 商业银行在全球金融机构 中仍然是当之无愧的霸主, 至2011年底, 商业银行总资产 130.23 万亿美元, 占GDP的 $217.73 \%$, 是影子银行总资产的 1.95倍。从2002-2011年商业银行和影子银行总量对比来 看, 全球大多数国家和地区仍然是以商业银行为主体的间 接融资体系，基于基础货币、货币乘数、广义货币量的传 统货币分析方法仍然有效, 同信用货币的扩张远超出实体 经济的增长, 2002年以来商业银行与GDP之比逐年上升, 尽管2010年和2011年有所回落, 但目前资产总量扩张趋势 并未改变。

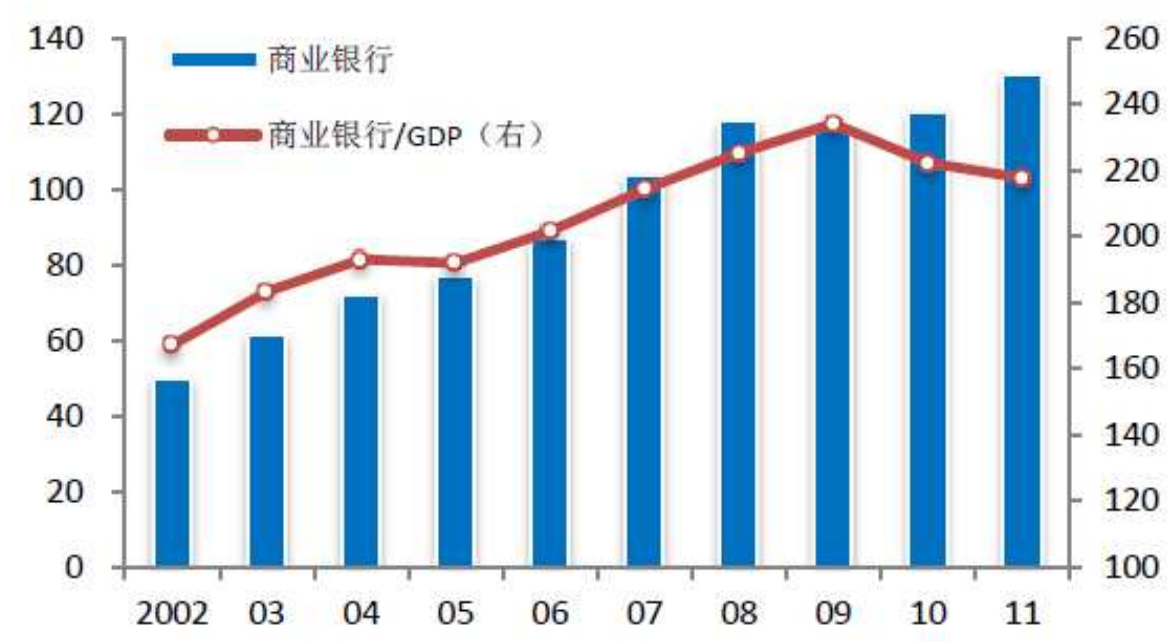

图1 商业银行总资产与GDP之比（万亿美元，\%）。 


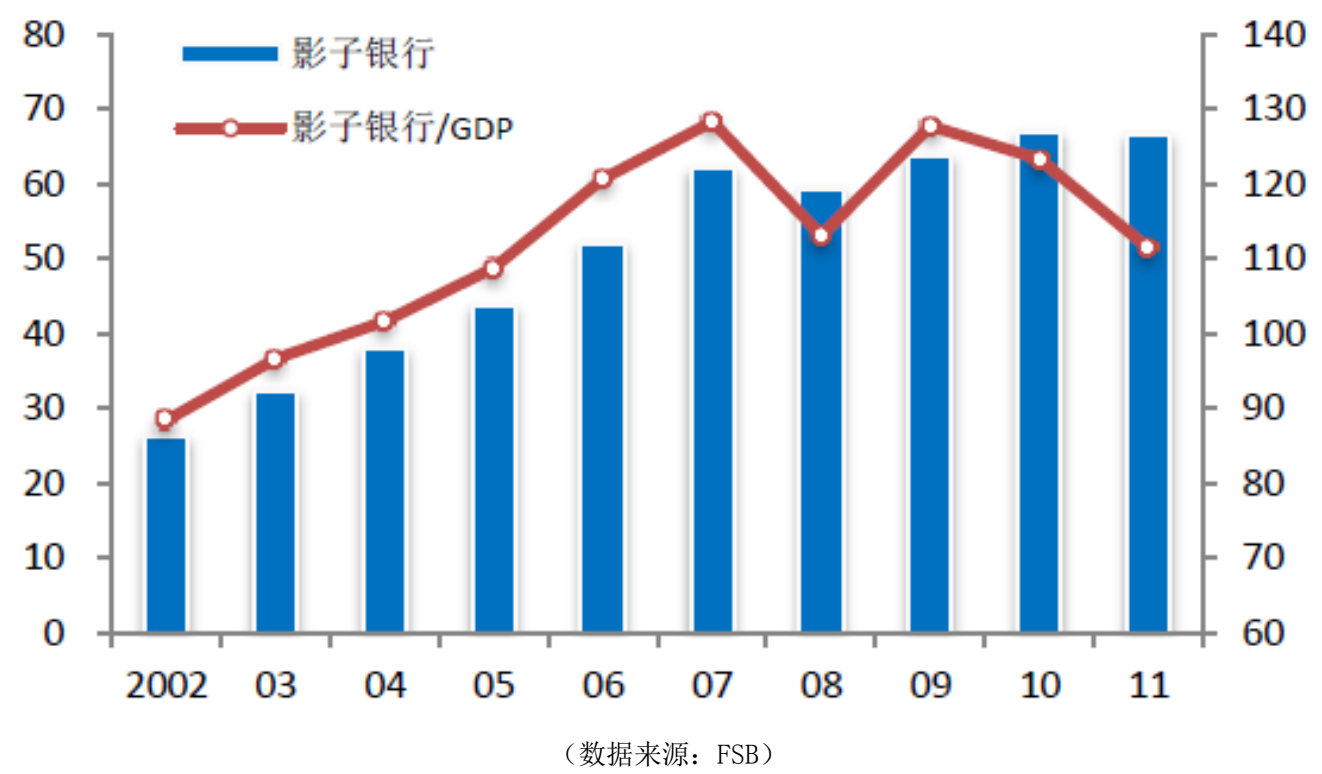

图2 影子银行总资产与GDP之比（万亿美元，\%)。

\subsection{2. 影子银行增长速度相较商业银行存在优势}

以2007 年为界大致可分为美国次贷危机前与危机后 时期, 对比影子银行与商业银行资产年均复合增长率, 尽 管两者在危机后的增速均有大幅下降, 但影子银行仍具有 比较优势, 其2002-2007年均复合增长率为 $18.65 \%$, 优于 商业银行的15. 74\%的增速；2008-2011年影子银行年均复 合增长率为 $3.92 \%$ ，仍高于商业银行 $3.29 \%$ 的增速。

\subsection{3. 影子银行总资产仍为少数国家和地区主导}

对于各国影子银行总资产占比，美国、欧元区、英 国和日本在全球范围内仍具有绝对优势, 至2011年底, 美国影子银行总资产达 23 万亿美元, 占 21 个国家和地 区全部影子银行资产的 $35 \%$, 占比较2005 年的44\%有所下 降; 欧元区影子银行总资产 22 万亿美元, 占比为 $33 \%$, 较 2005 年的 $31 \%$ 略有上升; 英国占比 $13 \%$, 较 2005 年的 $9 \%$ 大幅上升; 日本占比 $6 \%$, 较2005 年的 $7 \%$ 略有下降。这 四个国家和地区占比从2005 年的 $91 \%$ 下降为 2011 年的 $87 \%$, 总体而言在全球范围内的影子银行游戏中仍是绝对 主力。新兴市场影子银行发展迅速, 但总资产存量较少, 占比均处于较低的边缘区域, 至2011 年底, 韩国、巴西、 香港、新加坡、中国、印度占比仅为 $2 \% 、 2 \% 、 2 \% 、 1 \%$ 、 $1 \%$ 、 $1 \%$ 。

\section{4. 中国影子银行的比较分析}

\section{1. 中国影子银行体系的基本情况}

\section{1. 1. 中国影子银行体系的范围}

中国的信托公司、财务公司、小额贷款公司、汽车金 融公司、金融租赁公司、消费金融公司、典当行、民间信 用、农村资金互助社、地下钱庄等均不同程度上开展了影 子银行业务。虽然银监会认为, 以上的非银行金融机构已 纳入监管, 严格意义上不属于影子银行范畴。但中国的影
子银行体系的分类，是基于提供信用和监管套利的角度。 因此, 根据FSB的定义, 中国影子银行体系仍可分为三个 层面。

1. 银行的表外业务系统, 包括银行理财产品、委托贷 款。对于银行已承兑未贴现的汇票, 犹豫已计提风险资产, 不再纳入影子银行范畴。

2. 非银行类金融机构但具有信用创造能力的系统, 包 括信托、小额贷款、担保、融资租赁等公司。对已纳入央 行信贷口径统计的财务、银行系金融租赁、汽车金融等公 司予以扣除。

3. 广泛的民间借贷金融系统，包括商会、合会、互助 基金, 以及新兴的P $2 \mathrm{P}$ 网络信贷。

\section{1. 2. 中国影子银行体系的规模}

根据中国影子银行体系的划分, 各层面规模大致如下。

1. 银行的表外业务规模约为 10.3 万亿, 其中委托贷款 5.4 万亿, 银行理财产品 6.7 万亿, 需扣减银信合作 1.8 万 亿。

2. 非银行类金融机构规模约为 10.5 万亿，其中资金信 托 6.3 万亿，担保公司资本 0.6 万亿，典当余额 1.5 万亿、 小额贷款 0.5 万亿, 融资租赁 1.3 万亿, PE0. 2 万亿, REITs0.04万亿。

3. 民间融资规模约为 $3-5$ 万亿。根据央行民间融资调 查， 2011年5月末中国民间融资总量约为 3.38 万亿，银监 会对民间融资规模进行测算，同时，P2P模式的网络信贷 和理财公司也在迅速扩张。

因此, 预计中国影子银行体系规模约为 25 万亿, 与名 义 GDP 之比约为 $56 \%$, 与表内信贷之比约为 $41 \%$ 。尽管中国 影子银行规模与美国等国家和地区相比仍较小，但是其发 展的迅速和蕴藏的风险还是引起了监管的关注, 并且有可 能对金融市场造成一定的冲击。 


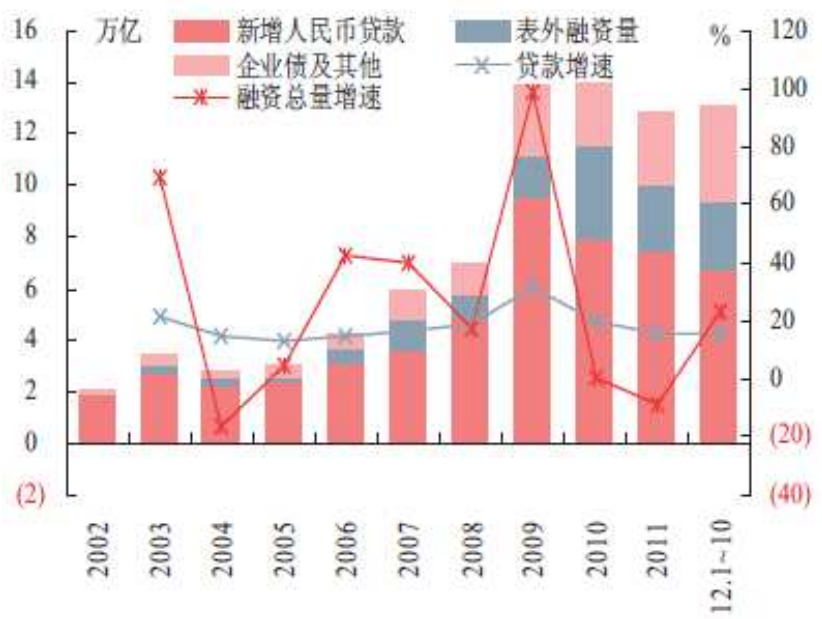

(数据来源: 央行网站)

图3 社会融资总量所反映的表位融资发展迅速。

\section{2. 中国影子银行体系分析}

\subsection{1. 中国影子银行快速发展原因}

近年来，中国的影子银行体系发展呈现蓬勃态势，有 以下主要原因。
1. 信贷作为配给资源的长期存在, 资本市场仍不发达, 银行体系的利率被压制, 实际贷款利率偏低, 导致信贷过 度需求, 不同信贷主体转而谋求非银行信贷。

2. 公众试图对抗高通货膨胀的不利影响和绕过利率 管制, 非正式的贷款和理财产品需求大增。

\subsection{2. 中国影子银行的信用扩张效应}

中国影子银行在机构运作上具备信用创造条件, 但是 中国融资市场以银行为主导, 加上资本市场发展并不成熟, 资产证券化机制不成熟, 所以影子银行信用创造功能并不 强大，但是仍存在信用扩张效应。

1. 银行表外业务系统。存款类机构的理财表外产品, 扩张了银行系统的融资规模; 投资银行机构的资产证券化、 信贷转让业务，扩展了银行的可贷资金。

2. 非银行金融机构系统。增信类机构, 直接增加了银 行系统的信贷扩张; 抵押类机构, 直接扩张非银行信贷; 直接信用，扩大了创业类企业的投资。

3. 民间金融系统。有组织信用转移机制，推进了信用 的派生创造; 信贷组织与信贷经纪人, 润滑了民间信用, 扩大了规模; 网络借贷电子平台、传媒通道, 解决了小型 经济活动主体的融资搜索难题; 工商企业钱庄化融资模式, 直接扩大了信用基础的投资与消费规模; 传统民间合作金 融, 发挥了支持弱势经济活动主体的融资功能。

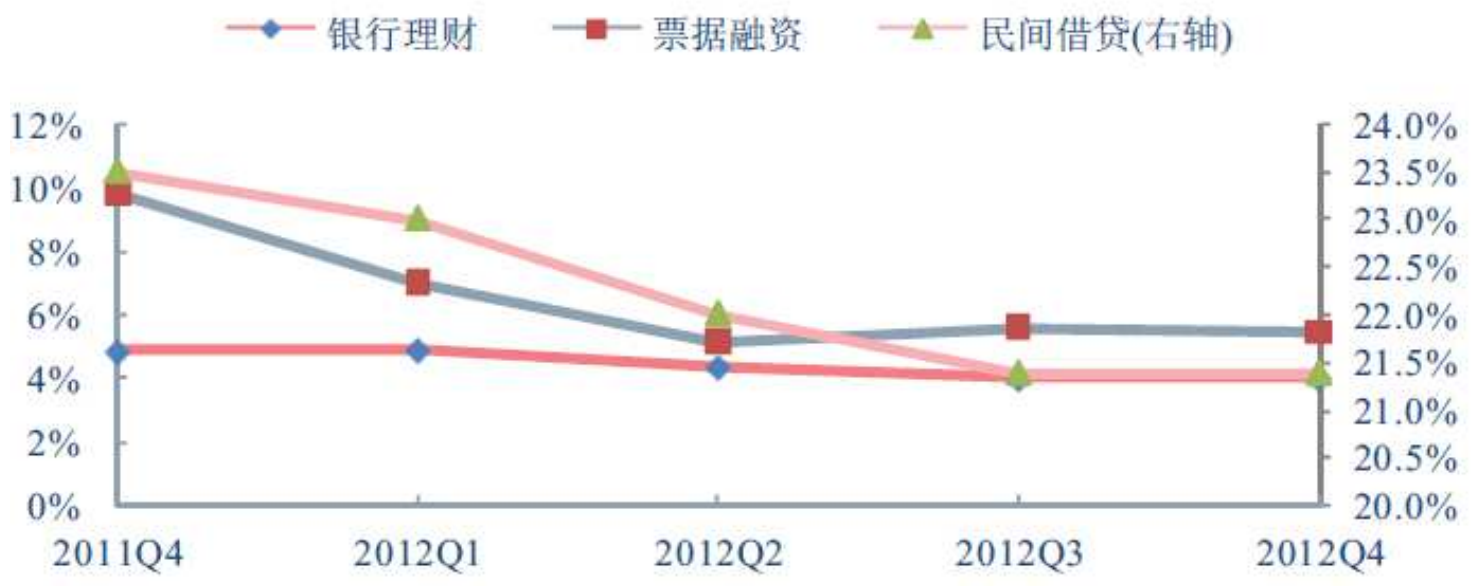

（数据来源：央行货币政策报告）

图4 影子银行体系收益率整体走低, 信用环境宽松。

\section{5. 结论}

影子银行作为游离于传统银行体系之外的信用中介, 能够参与信用、期限或流动性交换, 并进行信用创造。影 子银行具有期限错配、高杜杆性、对资产价格波动敏感、 较少受到监管的特征, 也增加了系统性的金融风险。根据 FSB评估，全球影子银行体系总规模67万亿美元，占金融 中介总资产的 $1 / 4$, 为商业银行规模的一半。美国具有最 庞大的影子银行规模, 约占全球影子银行总规模的 $35 \%$, 以金砖四国为代表的新兴市场国家也在金融危机后发展 迅速。
由于中国经济市场的特殊性, 中国影子银行的特征、 参与信用创造的过程和所面临的风险都有与国际影子银 行 (特别是以美国为代表的最大的影子银行体系) 有所不 同。所以中国影子银行虽然发展迅速, 但信用创造功能并 不大。

\section{参考文献}

[1] 杜亚斌, 顾海宁. 影子银行体系与金融危机 [J]. 审计与经济 研究, 2010, 25:82一87。 
[2] 何德旭, 郑联盛. 影子银行体系与金融体系稳定性 [J]. 经济 管理，2009 (11):20一 25。

[3] 刘文雯, 高平. “影子银行体系” 的崩塌对中国信托业发展 的启示 $[J]$.上海金融, 2010 (7):55一 59。

[4] 陈华, 刘宁. 影子银行助推金融危机的形成机理及前瞻性思 考 $[J]$. 新会计, $2010(11): 41$ 一 44 。

[5] 罗志华, 李耀深, 胡蓉. 银信合作: 制度约束下的金融创新 [J]. 西南金融, 2007，4:37一38。

[6] 文维虎, 陈荣. 重视影子银行动向, 避免风险隐患显现 [J]. 西南金融，2010(2):19一21。

[7] 辛乔利. 影子银行一揭秘一个鲜为人知的金融黑洞 [M]. 北 京:中国经济出版社，2010。
[8] Financial Crisis Inquiry Commission. Shadow banking and the financial crisis [M]. Financial Crisis Inquiry Commission, 2010, 5 .

[9] Gary Gorton, Andrew Metrick. Securitized banking and the run on repo[J]. Journal of Financial Economics . 2011 (3).

[10] Luca Dedola, Peter Karadi, Giovanni Lombardo. Global implications of national unconventional policies [J]. Journal of Monetary Economics. 2013 (1).

[11] NICOLA GENNAIOLI, ANDREI SHLEIFER, ROBERT W. VISHNY. A Model of Shadow Banking[J]. The Journal of Finance . 2013 (4). 